\title{
REVISITING MOBILE CROWDSENSING: AN OPEN CHALLENGE
}

\author{
Vittalis Ayu \\ Department of Informatics, Sanata Dharma University, Indonesia
}

\begin{abstract}
Mobile crowdsensing has become a new paradigm that enables citizens to participate in the sensing process by voluntarily gathering data from their smartphones to accomplish some given task. However, performing the sensing task generate lots of data resulting in various quality of the sensed data and high sensing cost in term of resource consumption. This matter became a significant concern in mobile crowdsensing as the mobile nodes which act as crowd sensors have limited resources. Moreover, an opportunistic mobile crowdsensing mechanism does not require user involvement, so the data collection process must be autonomous and intelligent to sense the data in the proper context. That is why context-awareness is also essential in opportunistic crowdsensing to maintain the sensed data quality. In this mini-review, we revisit the possibility of enhancing the mobile crowdsensing mechanism. We argue that improving the data collection process, including context-awareness, can optimize in-node data availability and sensed data quality. Besides, we also argue that finding optimization on inter-node data exchange mechanisms will increase the quality of the in-node data. Furthermore, smartphones that are related to humans as their owners reflect humans' physical and social behavior. We believe that considering contexts such as human social relationships and human mobility patterns can benefit the optimization strategies.
\end{abstract}

\section{KEYWORDS}

Mobile Crowdsensing, Data Quality, Context-awareness, Social Relation, Mobility Pattern.

\section{INTRODUCTION}

The emerging technology has enabled smartphones to enhance their connectivity and computing capabilities. Different sensors like gyroscope, accelerometer, camera, and microphone can be integrated easily into a single smartphone. This development empowers mobile devices to be able to sense, collect, and process information. Furthermore, sharing the collected information with nearby devices will form a collective knowledge about a particular phenomenon. This mechanism of sensing, collecting, processing, and sharing is called Mobile Crowdsensing (MCS). This term was first introduced by Ganti [1] as the collective ability to sense a kind of phenomenon from different devices.

A sensor-rich smartphone is a good upgrade for the existing environment-sensing mechanism. Furthermore, fast connectivity and various kind of sensors which already embedded in a mobile device can lead to lower deployment costs than those of the infrastructure-based sensing system. Ganti [1] observe the possibility of using sensing and connectivity capable devices, especially those that participate in the Internet of Things, to report their sensing result and form a collective knowledge. From then onwards, mobile crowdsensing has attracted many researchers to deepen their knowledge in this area.

David C. Wyld et al. (Eds): CMLA, CIoT, CSEIT, NCS, NLPD, SPM - 2021

pp. 93-101, 2021. CS \& IT - CSCP 2021

DOI: $10.5121 /$ csit.2021.111509 
In this mini-review, we intend to present the following aspects of MCS:

1. We provide an overview of MCS, existing implementation, and related research.

2. We investigate the existing implementation and research whether it has already incorporated social aspect and mobility model

3. We introduce potential challenge in MCS related to minimizing sensing and communication cost while maximizing data quality and a possibility to enhance it with the collaboration of social aspect and mobility model

\section{Overview of Mobile CrowdSensing}

The context scope in the sensing process is a crucial part of Mobile Crowdsensing. Lane [2] categorized mobile crowdsensing into two: personal and community sensing based on the scope. In personal sensing applications as depicted in Figure 1, the device user is the point of interest. For instance, monitoring and recognizing user-related posture and movement patterns for personal fitness $\operatorname{logs}$ or health care reasons. Community sensing as illustrated in Figure 2 monitors a particular phenomenon such as traffic congestion, pollution level or noise level that cannot be measured only by a single device. This phenomenon can be accurately measured when many observers such as stationery sensor, embedded sensors in mobile phone, car or bus sense the same phenomenon to acquire complete information.

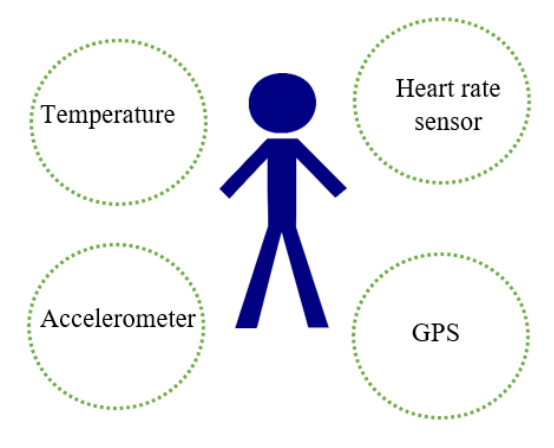

Figure 1. Personal sensing

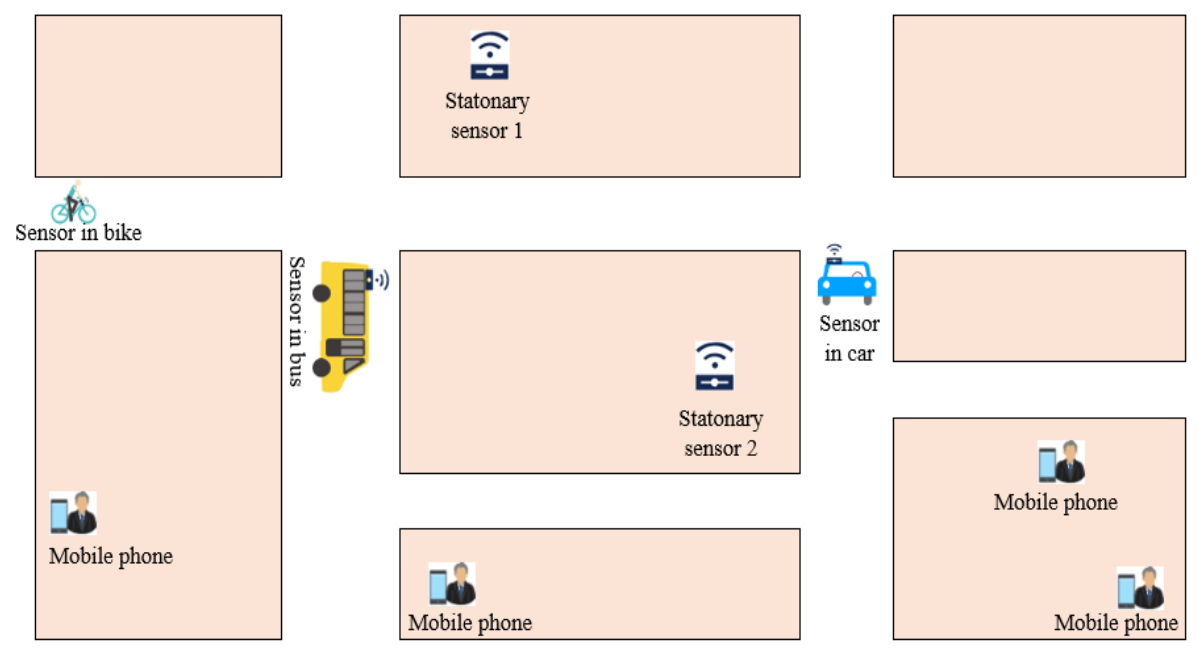

Figure 2. Community Sensing 
Due to the large-scale observation scope, there are two approaches to implement community sensing: participatory sensing and opportunistic sensing. The former required the user to register and thus be actively involved in the data sensing and collecting process, whereas the latter only needed passive participation. The participatory sensing process required awareness from the contributed citizen. The citizen as smartphone's owner has to define what, when dan was to sense a specific phenomenon. On the other hand, opportunistic sensing does not require user intervention as the data was collected automatically.

Consequently, opportunistic mobile crowdsensing relies heavily on the application to do the sensing task. Hence, this sensing mechanism needs to be autonomous and more intelligent since it has to recognize the sensing context on its own without the assistance of a human. Moreover, opportunistic sensing needs to sample data more frequently because of the user's passive participation and likely lower data quality due to context inaccuracy than participatory sensing. However, these data collection processes raise concerns about resources such as storage and energy. Meanwhile, as mobile devices' resources are limited, we must optimize resources while maximizing data quality and availability.

Crowdsensing management as depicted in Figure 3 includes three processes: data collection, data forwarding, and data analysis. The first process is data collection which aims to minimize the sensing cost of crowdsensors while maximizing the sensing coverage and quality. This process includes user recruitment and task allocation. The user recruitment concern is selecting the suitable crowd sensors for the sensing tasks, while task allocation assigns suitable tasks for crowdsensors. The second process is the data forwarding process which includes the communication aspect of the crowdsensing process. Lastly, the data analysis process aims to aggregate and extract the information from collected raw sensed data.
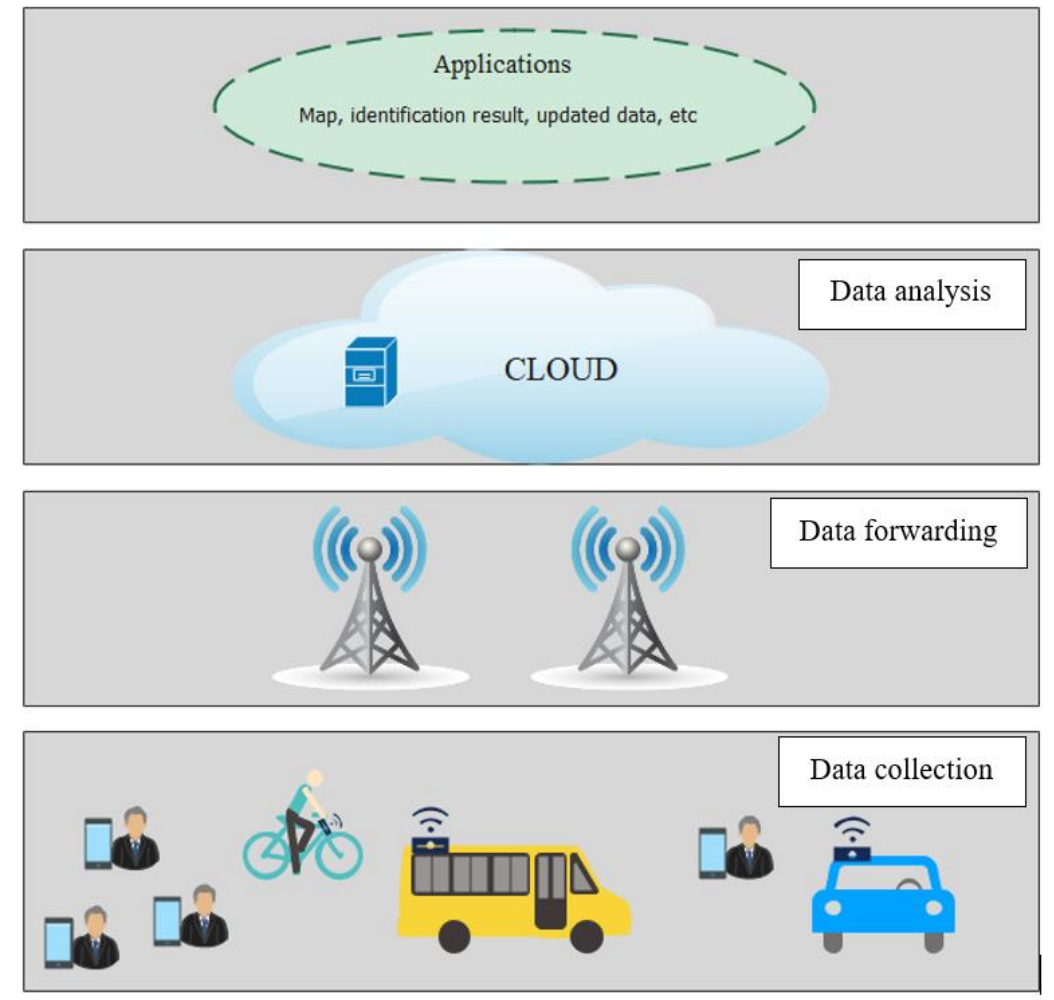

Figure 3. Crowdsensing management layer 
Crowdsensing applications are already implemented to build collective knowledge in some domains of interest, such as environment monitoring, e-commerce, and healthcare. Several studies [3][4][5][6] had already implemented the crowdsensing process to observe some aspects of the environment. Yan's research [3] relies on a driver's smartphone to monitor a traffic congestion situation and report it to the cloud-based server. After the cloud-based server receives the report, it aggregates the data and subsequently distributed the overall traffic condition to the other drivers who use the same application to be informed of the event and thus avoid the congested road. FixMyStreet [4] collects reports from citizens regarding some trouble related to the street and its supporting public facilities. Research conducted by El-Wakeel [5] monitors road conditions and road hazards by analyzing vehicle movement recorded by sensors both in the land vehicle and driver's smartphone. Air quality monitoring using SecondNose [6] had been done in Trento, Nothern Italy, with 80 participants on the data collection process. These participants collected air condition with sensors and aggregated the data. The smartphone's owner gets the complete information of the air quality in its surroundings (measured by the smartphone's sensors) and the overall air quality map generated by the aggregated data. In e-commerce, LiveCompare [7] uses participatory sensing to compare different prices from a specific product in different grocery stores. Abu-Elkheir [8] introduces crowdsensing as a means to get collective knowledge about an emergency. Healthcare framework AllergyMap [9] collect data from patients and can further visualize the distribution of different allergens and irritants concerning the spatial and temporal distribution.

These crowdsensing applications, as mentioned earlier, can be deployed with various kinds of underlying technologies. Capponi [10] differentiates the underlying of mobile crowdsensing technologies into two significant groups: infrastructure and infrastructure-less. Infrastructure technology refers to the need for data delivery infrastructures such as cellular or vast area networks. In contrast, the infrastructure-less technology depends on device-to-device (D2D) communication such as WiFi Direct and Bluetooth.

The availability of infrastructure will be a crucial point to define proper crowdsensing management. In infrastructure-based crowdsensing, crowdsensors upload the sensed data via infrastructure such as cellular networks to the remote server. Subsequently, data analysis will be performed in the remote server then a global view of data is achieved. Whereas in infrastructureless-based crowdsensing, the data aggregation will be done in a distributed environment within the crowd. Consequently, data analysis is performed locally and the global view of the sensed data can not be achieved,

\section{ISSUES IN MOBILE CROWDSENSING}

Generally, the more data can be collected in MCS; the more complete the knowledge will be. However, having a sufficient number of users to perform the sensing task can be a challenging problem. In the data collection process, some aspects concerned are data quality, sensing cost, and privacy guarantee. Even though a large crowd of participants is helpful to do the data collecting process because of its extensive sensing coverage, not all of the collected data have the same quality, yet the amount of generated data is enormous. Therefore, it is necessary to find optimization between the number of recruited participants and sensed data quality.

On the other hand, from the user's perspective, doing the sensing task can deplete its resources such as energy and storage. Consequently, users will not always be willing to cooperate in the sensing process. Hence, there must be some incentive mechanism to compensate the willingness of the user to contribute to the sensing process. Privacy also becomes a concern in the data collection process. The anonymity of user identity has to be guaranteed in the sensing process while still maintain user verification to avoid the threat from malicious users. 
Meanwhile, there are more to concern with the different perspectives of how users collect the data by participatory and opportunistic sensing. In participatory sensing, to get involved in the sensing process, the user's decision on how, what, when dan, where to sense is purely human's who act as the smartphone owner, thus indirectly including human's intelligence in the process. On the other hand, in opportunistic crowdsensing, the burden shifted to the application, which obliges intelligently aware of the sensing context hence makes context-awareness become an addition of data collection aspects to be concerned in opportunistic crowdsensing.

Data collection management in crowdsensing includes two processes: user recruitment and task allocation. The user recruitment process addresses the necessity in mobile crowdsensing to minimized the number of contributed users required in the sensing process while maintaining the sensing coverage and sensed data quality. On the other hand, the task allocation process concerns how to assign the right task to the suitable recruited user. Furthermore, those four aspects mentioned earlier (data quality, sensing cost, privacy, and context awareness) are partially addressed in the research in user recruitment and task allocation to improve the data collection process in mobile crowdsensing.

Research in [11][12] has been conducted to improve the user recruitment process. Wang [11] introduced PURE-DF to recruit participants in participatory crowdsensing. PURE calculate the estimation of the user's arrival in PoI and the user's subscription plan to minimize recruitment cost. Furthermore, Delegation Forwarding (DF) is used to optimize the trade-off between the delivery ratio of the sensed data and the number of required participants. Although this method can successfully minimize the recruitment cost and the number of recruited participants, this study assumes there is always sufficient bandwidth dan contact duration to successfully deliver the sensing task and exchange sensing data with other nodes. The Secure User Recruitment (SUR) protocol proposed by Xiao [12] uses a Greedy algorithm to recruit the nearly minimum user while ensuring the data quality and privacy using the semi-honest model. However, this mechanism assumes that the user mobility model is independent while the human mobility model is not fully independent. Besides smartphones, a vehicle with embedded sensors can act as crowd sensors. However, not all vehicles will voluntarily contribute to the sensing process. Research conducted by Liu [13] introduces an incentive mechanism to increase the participation of vehicles in the crowdsensing process.

To address task allocation, Capponi et al. 1 [14] introduce an energy-efficient data collection framework that can reduce the sensing cost and maximize the data quality considering the user's level of remaining energy and matching it with a suitable sensing task. The approach use context in the allocation process called Context-Aware Task Allocation (CATA) has been introduced by Hassani [15]. CATA measures similarity between task and user's context to compute the probability of successfully executing the task for a given user. When the probability of success is high, then the task will be allocated to the user.

\section{Research Challenges in Mobile Crowdsensing}

From those issues mentioned above, we found two challenges that can still be further studied: maximize data quality; minimize sensing and communication cost.

Maximize data quality. To maximize data quality, we need to find a suitable number of recruited users to perform the sensing task, hence covering a certain area. Furthermore, because we focus on opportunistic mobile crowdsensing, we need to improve the sensing process to be more intelligent. 
We argue that applying context-awareness will improve the data quality in the data collection process. In mobile crowdsensing, each node can do the local computation. Hence, the extraction of contextual information from raw sensed data can be performed inside the node itself. Hassani [15] implement context-awareness in the crowdsensing process by matching the task context with the participant context to allocate the suitable task to a certain participant. However, although the data were collected by smartphones, the collected data are then processed in a centralized server. The implementation on device to device communication network (D2D) and further use of distributed processing is possible. Artificial intelligence also can be utilized to derive the semantic characteristic of collected raw data. El-Wakeel [5] incorporate Support Vector Machine (SVM) to classify the collected data into eight different road anomaly contexts. Although the classification is done successfully, the diversity of sensors, vehicle's condition, and weather condition influence the classification process.

We also argue that involving the influence of mobility pattern of mobile nodes is advantageous to define which node to recruit based on spatial-temporal dimension because some people may regularly visit someplace after some interval. Moreover, we expect that considering the mobility pattern of the mobile node will benefit the context inference of the crowdsensing process. The mobility models presented in Table 1 already captured some mobility models used in earlier experiments. There is much diversity of mobility models such as synthetic mobility, real-world, and random mobility. However, none of these models are used to define the overlay social characteristics derived from the participant's movement.

Table 1. Summary of Mobile Crowdsensing Existing Solutions

\begin{tabular}{|c|c|c|c|c|c|c|c|c|}
\hline \multirow[b]{2}{*}{ Ref } & \multicolumn{2}{|c|}{ Participation } & \multicolumn{2}{|c|}{ Underlying technologies } & \multirow{2}{*}{$\begin{array}{l}\text { Data } \\
\text { quality }\end{array}$} & \multirow{2}{*}{$\begin{array}{l}\text { Sensing } \\
\text { cost }\end{array}$} & \multirow{2}{*}{$\begin{array}{l}\text { Social } \\
\text { aspect }\end{array}$} & \multirow{2}{*}{$\begin{array}{l}\text { Mobility model and } \\
\text { geographical scope }\end{array}$} \\
\hline & $\begin{array}{l}\text { Participato } \\
\text { ry }\end{array}$ & Opportunistic & $\begin{array}{l}\text { Infrastruct } \\
\text { ure }\end{array}$ & $\begin{array}{l}\text { Infrastructure- } \\
\text { less }\end{array}$ & & & & \\
\hline [3] & $\checkmark$ & - & $\checkmark$ & - & - & $\checkmark$ & - & $\begin{array}{l}\text { real vehicle movement of } \\
\text { Guangzhou City }\end{array}$ \\
\hline [4] & $\checkmark$ & - & $\checkmark$ & - & - & - & - & geographical region of Brussel \\
\hline [5] & - & $\checkmark$ & - & $\checkmark$ & $\checkmark$ & - & - & $\begin{array}{l}\text { vehicle movement in Kingston, } \\
\text { Canada }\end{array}$ \\
\hline [6] & - & $\checkmark$ & - & $\checkmark$ & - & - & - & human real movement in Trento \\
\hline [7] & $\checkmark$ & - & $\checkmark$ & - & $\checkmark$ & - & - & $\begin{array}{l}\text { Geographical region of Durham, } \\
\text { North Carolina }\end{array}$ \\
\hline [8] & $\checkmark$ & - & - & $\checkmark$ & $\checkmark$ & - & $\checkmark$ & $\begin{array}{l}\text { drones movement based on } \\
\text { roads }\end{array}$ \\
\hline [9] & $\checkmark$ & - & $\checkmark$ & - & - & $\checkmark$ & - & geographical region of Greece \\
\hline [11] & $\checkmark$ & - & $\checkmark$ & - & - & $\checkmark$ & - & $\begin{array}{l}\text { roma/taxi trace set, epfl trace } \\
\text { set, geolife trace set }\end{array}$ \\
\hline [12] & $\checkmark$ & - & - & $\checkmark$ & $\checkmark$ & - & - & synthetic mobility model \\
\hline [13] & - & $\checkmark$ & $\checkmark$ & - & - & $\checkmark$ & - & $\begin{array}{l}\text { crawdad dataset of taxis in San } \\
\text { Fransisco }\end{array}$ \\
\hline [14] & - & $\checkmark$ & $\checkmark$ & - & $\checkmark$ & $\checkmark$ & - & $\begin{array}{l}\text { pedestrian mobility in } \\
\text { Luxemburg }\end{array}$ \\
\hline [15] & - & $\checkmark$ & $\checkmark$ & - & $\checkmark$ & $\checkmark$ & - & synthetic mobility model \\
\hline
\end{tabular}

Besides, we believe that data quality can be further refined by the data aggregation process facilitated by an inter-node data exchange mechanism. While inspecting other node's data, we may found similar data with different quality. Hence, we can compare these data and choose which data to keep.

In addition, different mobility characteristics of the nodes can be considered in the sensing process related to the coverage and density of the sensing area. For example, people who tend to travel in the more extensive coverage area can most likely sense a lot of different data. However, 
the communication cost will be huge as the assigned task will rarely match with the in-node available data.

Also, people whose mobility patterns covered a dense area tend to have more similar data with other people compared to people whose mobility patterns covered a sparse area. When people arrive in a dense area, she/he can drop the data more frequently as another node may have similar data.

Minimize sensing and communication cost. Other than minimizing sensing and communication costs, there are factors to consider, such as the underlying communication technology and resource allocation. We focus on inspecting the scenario of infrastructure-less scenario. D2D communication over Mobile Adhoc Network (MANET) and Opportunistic Network (OppNet) are the two candidates for the underlying technology of the crowdsensing process in the D2D scenario. Although these two underlying communication technologies are the basis of mobile adhoc communication, they differ in some aspects. Even though node mobility in MANET is dynamic, there is always an end-to-end path between these nodes, so the global knowledge is available in MANET. In contrast, in the opportunistic network, there are not.

On the other hand, in OppNet, there are intermittent connectivity and opportunistic encounter between nodes. Thus, the end-to-end path does not exist. Moreover, the contact duration between nodes and encounter probability of nodes varies. Consequently, data aggregation and data forwarding in this network have to be distributed because of the absence of global knowledge of the network. However, the opportunistic network will benefit the crowdsensing process because of the availability of bundle layers inside the nodes, which enable the store-carry-forward mechanism. In an opportunistic network, data can be carried around even when the end-to-end path does not exist. This behavior will reduce the communication cost as the data can be carried around between the nodes themselves without infrastructure involvement. Researchers in $[5][6][8][12]$ are already conducted in infrastructure-less settings. However, these four researchs still incorporate edge cloud in the data aggregation process.

Because of the limitation of mobile node resources such as storage and energy, we argue that resource-aware data collection and forwarding are essential to reduce the sensing cost. The willingness to participate in the crowdsensing process must be compensated with some reward from the incentive mechanism. In future research, a game theory-based incentive mechanism can be utilized to optimize the resource and the willingness to cooperate. The reputation-based reward can benefit the mechanism as the incentive scheme does not have to be in monetary forms. Although research in [9][11][13][14][15] implemented an incentive mechanism to encourage participation and minimize resource consumption, to the best of our knowledge, there are none that corporate the derivation of the social context of the collaborating nodes in the opportunistic mobile crowdsensing. Elkheir's research [8] infuses social media to collect emergency data. However, the overlay social characteristics such as community and social ties of the collaborating nodes have not been explored yet. There are some efforts in [16][17][18] to incorporate social aspects of the routing process in the opportunistic network, such as similarity and centrality.

\section{CONClusion}

The emerging technology has enabled the mobile crowdsensing paradigm to form collective knowledge within the crowd, with some aspects to be concerned, such as sensed data quality and sensing cost. Furthermore, in opportunistic mobile crowdsensing, context-awareness is essential because the sensing mechanism needs to be intelligent as the sensing process does not include active human participation. As the opportunistic network will serve as the underlying 
communication network, the intermittent connectivity, and various contact opportunity, the crowdsensing mechanism should employ a distributed algorithm which can be later implemented in this network. Therefore, mobile crowdsensing still faces open challenges to maximize the sensed data quality while minimizing the sensing cost. Moreover, incorporating social aspects and mobility patterns as additional aspects is a possible thing to be done in future studies.

\section{REFERENCES}

[1] R. K. Ganti, F. Ye and H. Lei, (2011) "Mobile crowdsensing: current state and future challenges", IEEE Communications Magazine, vol. 49, no. 11, pp. 32-39.

[2] N. D. Lane, E. Miluzzo, H. Lu, D. Peebles, T. Choudhury \& A. T. Campbell, (2010) "A survey of mobile phone sensing", IEEE Communications Magazine, vol. 48, no. 9, pp. 140-150.

[3] Hehua Yan, Qingsong Hua, Daqiang Zhang, Jiafu Wan, Seungmin Rho, \& Houbing Song (2017) "Cloud-Assisted Mobile Crowd Sensing for Traffic Congestion Control", Mobile Netw Appl 22, 1212-1218

[4] Pak, Burak, Alvin Chua \& Andrew Vande Moere. (2017) "FixMyStreet Brussels: SocioDemographic Inequality in Crowdsourced Civic Participation", Journal of Urban Technology, 24:2, 65-87

[5] A. S. El-Wakeel, J. Li, A. Noureldin, H. S. Hassanein \& N. Zorba, (2018) "Towards a Practical Crowdsensing System for Road Surface Conditions Monitoring", IEEE Internet of Things Journal, vol. 5, no. 6, pp. 4672-4685.

[6] Chiara Leonardi, Andrea Cappellotto, Michele Caraviello, Bruno Lepri, \& Fabrizio Antonelli, (2014) "SecondNose: an air quality mobile crowdsensing system", Proceedings of the 8th Nordic Conference on Human-Computer Interaction: Fun, Fast, Foundational (Nordi CHI '14), Association for Computing Machinery, New York, NY, USA, 1051-1054.

[7] Linda Deng \& Landon P. Cox, (2009) "LiveCompare: grocery bargain hunting through participatory sensing", Proceedings of the 10th workshop on Mobile Computing Systems and Applications (HotMobile '09). Association for Computing Machinery, New York, NY, USA, Article 4, 1-6.

[8] M. Abu-Elkheir, H. S. Hassanein \& S. M. A. Oteafy, (2016) "Enhancing emergency response systems through leveraging crowdsensing and heterogeneous data," International Wireless Communications and Mobile Computing Conference (IWCMC), Paphos, Cyprus, pp. 188-193,

[9] L. A. Kalogiros, K. Lagouvardos, S. Nikoletseas, N. Papadopoulos \& P. Tzamalis, (2018) "Allergymap: A Hybrid mHealth Mobile Crowdsensing System for Allergic Diseases Epidemiology : a multidisciplinary case study," IEEE International Conference on Pervasive Computing and Communications Workshops (PerCom Workshops), Athens, Greece, pp. 597-602.

[10] A. Capponi, C. Fiandrino, B. Kantarci, L. Foschini, D. Kliazovich \& P. Bouvry, (2019) "A Survey on Mobile Crowdsensing Systems: Challenges, Solutions, and Opportunities", IEEE Communications Surveys \& Tutorials, vol. 21, no. 3, pp. 2419-2465.

[11] E. Wang, Y. Yang, J. Wu, W. Liu \& X. Wang, (2018) "An Efficient Prediction-Based User Recruitment for Mobile Crowdsensing", IEEE Transactions on Mobile Computing, vol. 17, no. 1, pp. $16-28$.

[12] M. Xiao, J. Wu, S. Zhang \& J. Yu, (2017) "Secret-sharing-based secure user recruitment protocol for mobile crowdsensing", IEEE INFOCOM 2017 - IEEE Conference on Computer Communications, Atlanta, GA, USA, pp. 1-9.

[13] L. Liu, X. Wen, L. Wang, Z. Lu, W. Jing \& Y. Chen, (2020) "Incentive-Aware Recruitment of Intelligent Vehicles for Edge-Assisted Mobile Crowdsensing", IEEE Transactions on Vehicular Technology, vol. 69, no. 10, pp. 12085-12097.

[14] A. Capponi, C. Fiandrino, D. Kliazovich \& P. Bouvry, (2017) "Energy efficient data collection in opportunistic mobile crowdsensing architectures for smart cities," IEEE Conference on Computer Communications Workshops (INFOCOM WKSHPS), Atlanta, GA, pp. 307-312.

[15] A. Hassani, P. D. Haghighi \& P. P. Jayaraman, (2015) "Context-Aware Recruitment Scheme for Opportunistic Mobile Crowdsensing", IEEE 21st International Conference on Parallel and Distributed Systems (ICPADS), Melbourne, VIC, Australia, pp. 266-273.

[16] P. Hui, J. Crowcroft \& E. Yoneki, (2011) "BUBBLE Rap: Social-Based Forwarding in DelayTolerant Networks", IEEE Transactions on Mobile Computing, vol. 10, no. 11, pp. 1576-1589. 
[17] E. M. Daly \& M. Haahr, (2009) "Social Network Analysis for Information Flow in Disconnected Delay-Tolerant MANETs", IEEE Transactions on Mobile Computing, vol. 8, no. 5, pp. 606-621.

[18] D. Rothfus, C. Dunning \& X. Chen, (2013) "Social-similarity-based routing algorithm in Delay Tolerant Networks", IEEE International Conference on Communications (ICC), Budapest, Hungary, 2013, pp. 1862-1866.

\section{AUTHORS}

Vittalis Ayu received the Bachelor degree in Computer Engineering from Telkom University and Master degree in Computer Science from Gadjah Mada University. She is currently a Lecturer in Department of informatics, Sanata Dharma University, Yogyakarta, Indonesia. Her research interest includes computer network, distributed network, mobility model, Internet of Things and mobile crowdsensing.

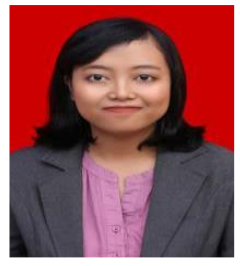

(C) 2021 By AIRCC Publishing Corporation. This article is published under the Creative Commons Attribution (CC BY) license. 